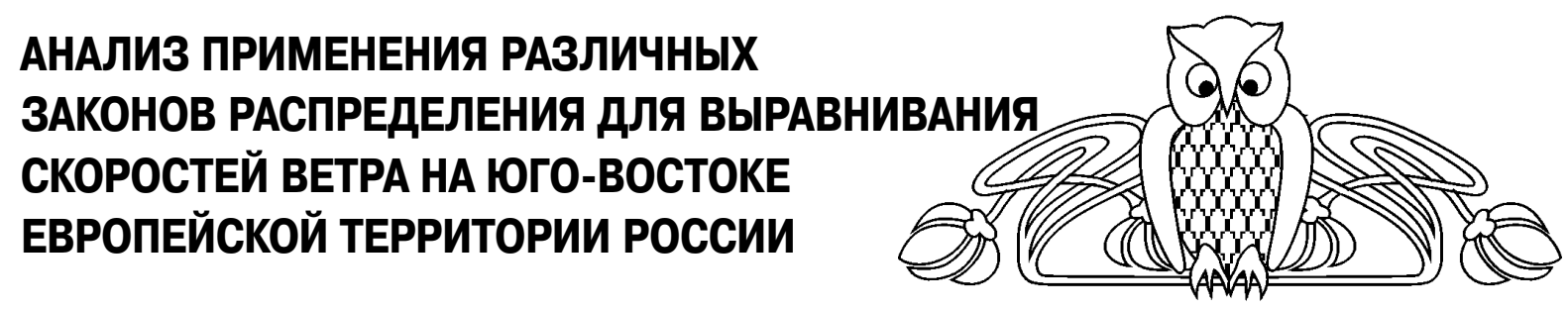

\section{А.Б. Рыхлов}

Саратовский государственный университет, кафедра метеорологии и климатологии E-mail: kafmeteo@mail.ru

Проведен статистический анализ наиболее часто используемых законов распределения, применяемых для расчета вероятности различных скоростей ветра: Пуассона, нормального, логнормального и Вейбулла-Гудрича - с применением критериев Пирсона и Колмогорова. Выявлено, что неоспоримым преимуществом по двум указанным критериям обладает распределение Вейбулла-Гудрича. Этот закон рекомендуется использовать для математического описания ветрового режима юго-востока европейской территории России.

Ключевые слова: климат, скорость ветра, распределение, закон распределения, погрешности, моделирование ветрового режима.

\section{Analysis of Different Distribution Laws Using for Wind Speed Leveling on the South-West of European Part of Russian Federation}

\section{A.B. Rikhlov}

Statistical analysis of the most popular and often used laws of distribution, that used for probability estimation of various wind speed: Puasson, normal, longnormal and Viebulla-Gudrich laws with application of Pirson and Kolmogorov criteria was carried out. This law is recommended to use for wind conditions mathematical description for on the south-west of european part of Russian Federation.

Key words: climate, wind speed, distribution, distribution law, errors, wind condition modeling.

В метеорологии с очень давних времен делались попытки вывести «всеобщий» закон распределения скоростей ветра. Еще в 1889 г. Б.И. Срезневский [1] показал возможность применения к описанию распределения скоростей ветра у поверхности земли закона Максвелла, известного в кинетической теории газов. Позднее неоднократно повторялись попытки найти теоретическим путем такое распределение ветра, которое совпадало бы с наблюдаемым [2-4]. В постановке этой задачи выбирались разные масштабы осреднения. Они были небольшими при изучении порывистости и значительными при изучении климатических особенностей.

К сожалению, в выполненных многочисленных работах, несмотря на математическую их строгость, не всегда производилось сравнение с наблюдающимся в природе ветровым режимом.
Естественно, результаты этих работ не внедрены в метеорологическую практику. Некоторым исключением являются работы английских метеорологов Брукса и Карузерс, которые применили статистические законы распределения ветра к климатологии ветра верхних уровней [5]. В настоящее время в ряде научных публикаций имеются указания, что распределение скоростей ветра по всей совокупности наблюдений может успешно описываться нормальным и логнормальными законами распределения, а также распределениями Пуассона и Вейбулла. На основе положений статистики нами проведено исследование соответствия этих теоретических законов распределений эмпирическому ветровому режиму на рассматриваемой территории с целью выявления наиболее оптимального.

На основании изучения распределения ветров И.Г. Гутерманом [6] показывается, что к климатическому распределению скоростей в свободной атмосфере с успехом может быть применен закон распределения Максвелла. В дифференциальной форме он может быть записан следующим образом:

$$
f(v)=\frac{\pi v}{2 \bar{v}^{2}} e^{-\frac{\pi v^{2}}{4 \bar{v}^{2}}}
$$

или в интегральной форме

$$
F(v)=e^{-\frac{\pi v^{2}}{4 \bar{v}^{2}}}
$$

где $v$ - индивидуальное значение модуля скорости ветра; $\bar{v}$ - средняя многолетняя скорость (т.е. среднее из всех значений модулей векторов ветра за период осреднения); $f(v)$ - повторяемость или дифференциальная вероятность; $F$ - интегральная вероятность (обеспеченность). Для определения $F(v)$ требуется знать лишь среднюю скорость $\bar{v}$. В аэроклиматологии принято характеризовать поле скорости ветра даже при наличии ряда 5-10-летней продолжительности. При этом вычисляются средняя скорость ветра $\bar{v}$ и повторяемость скоростей различных значений по градациям.

Для наземных наблюдений по флюгеру идея применения распределения Максвелла позднее была оставлена. Опытные данные не подтвердили соответствие фактического распределения теоретическому, главным образом из-за увеличенного числа штилей у поверхности земли. Однако простота восстановления режима ветра всего лишь 
по средней скорости приводит к использованию его при решении отдельных задач и сегодня. Применение законов распределения ветра для наблюдений за ним на высоте флюгера подробно анализируется в работе Гриневича [3]. Исследованием закономерностей распределения ветра также занимались Хессельберг [7], Кузнецов [8], Омшанский [9].

Выравнивание распределений скоростей ветра - нетривиальная задача. Подбор закона распределения зависит от многих факторов, в том числе и от поставленной задачи. Для определения расчетных скоростей ветра редкой повторяемости, в частности максимальных скоростей ветра, возможных в той или иной местности, основным требованием является надежное совпадение эмпирических и теоретических распределений в области высоких значений. Вид закона распределения зависит и от используемой совокупности наблюдений. В частности, исходной совокупностью могут служить срочные наблюдения исследуемой характеристики за какой-либо период или же выборочные месячные и годовые максимумы. В результате исследований было высказано мнение, что если привлечена вся совокупность наблюдений, наиболее оптимальным является закон распределения Вейбулла.

Двухпараметрический закон Вейбулла-Гудрича имеет вид

$$
F(V>v)=\exp \left(-\frac{v}{\beta}\right)^{\gamma},
$$

где $F$ - функция распределения (обеспеченности) скорости ветра; $\beta$ - масштабный множитель, близкий к средней скорости ветра; $\gamma$ - параметр, характеризующий форму распределения.

Такая аппроксимация применительно к распределению скорости ветра впервые была предложена советскими учеными и широко использовалась для статистической экстраполяции максимальных скоростей ветра. Впоследствии аппроксимация распределения скорости ветра законом Вейбулла-Гудрича использовалась для той же цели рядом авторов $[10,11,12]$.

К сожалению, возможности, вытекающие из применения аппроксимации распределения скорости ветра законом Вейбулла-Гудрича, использованы далеко не полностью. Более того, параметры этого распределения не нашли отражения в $[13,14]$. Наиболее массовые расчеты были проведены лишь С.Д. Кошинским [10], который выполнил и ряд методических проработок, показав преимущества упомянутой аппроксимации. В этом отношении определенный интерес представляют работы зарубежных исследователей, которые, в частности, использовали указанную аппроксимацию для оценки ветроэнергоресурсов на территории США [15].

В климатологической практике для теоретического выравнивания скоростей ветра в свое вре- мя достаточно часто использовался нормальный (гауссовский) закон распределения. Вообще он является предельным для многих других теоретических распределений и образуется тогда, когда каждое значение случайной величины можно рассматривать как сумму большого числа независимых величин. Для надежной оценки параметров данного распределения требуется большой объем выборки. Плотность вероятности нормального закона определяется двумя параметрами: математическим ожиданием и дисперсией. Он задается для плотности и функции распределения выражениями:

$$
f(v)=\frac{1}{\sigma \sqrt{2 \pi}} e^{-\frac{(v-\bar{v})^{2}}{2 \sigma^{2}}}
$$$$
\text { или } F(V<v)=\frac{1}{\sigma \sqrt{2 \pi}} \int_{-\infty}^{v} e^{-\frac{(v-\bar{v})^{2}}{2 \sigma^{2}}} \text {, }
$$

где $\bar{v}$ и $\sigma$ - параметры распределения - среднее значение и среднее квадратическое отклонение скорости ветра. Для расчетов по этим формулам, прежде всего, вводят новую переменную $t$, которая рассчитывается по формуле

$$
t=\frac{x-\bar{x}}{\sigma} .
$$

Такой прием часто используется в климатических расчетах. Он удобен тем, что позволяет свести размерную случайную величину со средним значением $\bar{v}$ и средним квадратическим отклонением $\sigma$ к безразмерной величине $t$. Для случайной величины $t$ существуют статистические таблицы, содержащиеся во многих учебниках по теории вероятностей и математической статистике. Они значительно облегчают вычисления.

При использовании повторяемостей скорости ветра по градациям значений следует иметь в виду, что внутри градаций они могут быть распределены неравномерно, особенно для редких значений. В случае использования этих данных, по мнению Л.Л. Брагинской [12], М.В. Завариной [16], Л.Е. Анапольской и Л.С. Гандина и др. [17] приемлемыми законами распределения являются логнормальный, Максвелла, Вейбулла-Гудрича. Имеются работы, в которых утверждается, что распределение метеорологических элементов, имеющих физический предел с одной стороны (таких как количество осадков, высота нижней границы облачности и скорость ветра), хорошо описывается логнормальным распределением.

В отличие от нормального, логнормальное распределение формируется тогда, когда каждое значение случайной величины можно рассматривать не в качестве суммы, а как произведение большого количества случайных величин. В этом случае нормально распределяется логарифм случайной величины. 
Функция логнормального распределения имеет вид

$$
F(V<v)=\frac{1}{\sigma_{\lg v} \sqrt{2 \pi}} \int_{-\infty}^{\lg v} \mathrm{e}^{-\frac{(\lg v-\overline{\lg v})^{2}}{2 \sigma_{\lg v}^{2}}}
$$

Для того чтобы воспользоваться этим выражением, надо определить его параметры $\overline{\lg v}$ и $\sigma_{l g} v$.

При выборе типа уравнения распределения обычно преследуют две цели: с одной стороны, стремятся увеличить точность интерполяции эмпирических распределений, с другой - уменьшить объем вычислительных работ, связанных с определением параметров уравнения. В результате обработки данных наблюдений получают эмпирическую зависимость распределения, которая характеризует частоту различных значений исследуемой величины. Эмпирическое распределение всегда получается на основании ограниченного числа наблюдений и, как правило, не позволяет объективно судить о законе, которому подчиняется распределение генеральной совокупности значений изучаемой величины. Для установления такого закона обычно вводится предположение (гипотеза), что статистический ряд, лежащий в основе эмпирического распределения, представляет собой случайную выборку из генеральной совокупности, имеющей закон распределения $f(v)$. Параметры этой функции, являющейся теоретическим законом распределения, как уже говорилось, вычисляют по данным наблюдений. Естественно, что между эмпирическим и теоретическим рядами распределения всегда будет некоторое расхождение.

Возникает вопрос, можно ли получившиеся расхождения считать несущественными, имеющими случайный характер, обусловленный ограниченным объемом выборки, или же они являются существенными и противоречат гипотезам, согласно которым данный статистический ряд можно рассматривать как выборку из генеральной совокупности с принятым теоретическим законом распределения? Задача, таким образом, сводится к тому, чтобы установить, носит ли расхождение случайный характер или нет.

Для решения указанной задачи необходимо, прежде всего, выбрать оправданную меру расхождения между теоретическим и эмпирическим рядами. Оценка согласия между эмпирическим и теоретическим распределениями осуществляется на основании принятой гипотезы о случайном характере расхождения. В результате чего мера расхождения является случайной величиной, для которой может быть найден интегральный закон распределения. Далее пользуются «принципом практической невозможности маловероятных событий». Согласно этому принципу событие, обладающее малой вероятностью, считается практически невозможным. Поэтому если оно все же наблюдается, то это говорит о несостоятельности гипотезы, на основании которой была определена его вероятность. Для практического применения данного принципа необходимо задаться уровнем значимости $\left(P_{\mathrm{o}}\right)$ - это вероятность принятия случайного расхождения за систематическое. Сам выбор величины уровня значимости является условным. Чаще всего принимают $P_{\mathrm{o}}=0,05=5 \%$.

Существует ряд критериев согласия, основанных на изучении распределения различным образом составленной меры расхождения. В климатологической практике наиболее широко используются критерии Пирсона и Колмогорова. Но ни один из критериев согласия не может рассматриваться как достаточный, ведь в его основе лежит условно установленная мера расхождения и известная погрешность в оценке практически невозможных событий. Поэтому необходимо производить оценку по крайней мере по двум критериям согласия.

За меру расхождения теоретического и эмпирического распределений Пирсон принимает величину $\chi^{2}$, определяемую формулой

$$
\chi^{2}=\sum \frac{\left(\frac{m_{i}}{n}-p_{i}\right)^{2}}{p_{i}}
$$

где $n$-объем совокупности; $\frac{m_{i}}{n}$-относительные частоты эмпирического распределения; $p_{i}$ - соответствующие теоретические относительные частоты.

Для случайной величины $\chi^{2}$ был найден интегральный закон распределения:

$$
P\left(\chi^{2}>\chi_{0}^{2}\right)=1-F\left(\chi_{0}^{2}, g\right),
$$

где $g$ - число степеней свободы. Число степеней свободы вычисляется по формуле

$$
g=s-1,
$$

где $s-$ число градаций.

Если между теоретическими и опытными частотами существуют некоторые дополнительные связи, число которых $l$, то

$$
g=s-1-l .
$$

Так, если при расчетах частот по нормальному закону распределения $\bar{v}$ и б оценивались по опытным данным, то этим устанавливаются два дополнительных соотношения. В таком случае $l=2$, а $g=s-3$. Если $H_{0}-$ нулевая гипотеза, состоящая в том, что с вероятностью $99 \%$ результаты измерения скорости ветра согласуются с функци- 
ей распределения, то при $\chi^{2} \leq \chi_{0,99}^{2}$ гипотеза $H_{0}$ принимается, в противном случае отвергается.

А.Н. Колмогоров предложил другой критерий согласия, который в отличие от критерия Пирсона основан на сравнении интегральных законов опытного и теоретического распределений. В качестве меры расхождения А.Н. Колмогоров использует величину

$$
\lambda=D \sqrt{n}
$$

где $n$ - объем совокупности; $D$ - соответствует наибольшему расхождению теоретической и эмпирической обеспеченностей. Согласно теореме Колмогорова для различных уровней значимости $P_{0}$ можно рассчитать те значения $\lambda_{0}$, которые не должна превышать полученная из опыта величина $\lambda$. Практическое применение критерия $\lambda$ для оценки степени близости экспериментального и теоретического распределений сводится к следующему. Задавшись уровнем значимости $P_{0}=5 \%$, по специальным таблицам ((2)) определяют допустимое значение критерия согласия $\lambda_{0}$. Затем по данным теоретического и статистического рядов вычисляют величины различий, определяют наибольшую из них и оценивают значение $\lambda$. Если найденное таким образом $\lambda$ окажется больше $\lambda_{0}$, то при данном уровне значимости гипотеза должна быть отвергнута. В противном случае между теоретическим и эмпирическим распределениями существует удовлетворительное согласие.

Важным вопросом при рассмотрении теоретического и эмпирического распределений является определение точности расчета статистических характеристик. Если их точность не оценена или оценена неправильно, использование ее в климатических исследованиях может привести к неверным выводам. Рассматривая статистическую совокупность значений метеорологических величин как выборку из генеральной совокупности, статистические ошибки климатических характеристик оценивают по отношению к соответствующим характеристикам генеральной совокупности (неявно предполагаем, что нет закономерных изменений климата).

Средние погрешности климатических характеристик определяются средними квадратическими отклонениями этих характеристик из выборочных совокупностей данного объема от соответствующих характеристик генеральной совокупности. Если сделать допущение о стационарности метеорологических процессов и отсутствии внутрирядной связности, то формулу средней ошибки относительной частоты данной градации можно записать следующим образом:

$$
\sigma_{p} \approx \sqrt{\frac{P(1-P)}{n}},
$$

где $n$ - объем совокупности; $P$ - эмпирическая повторяемость скоростей ветра.

Следуя изложенным выше подходам и принципам, выработанным российской и зарубежной наукой, нами впервые решена задача выбора наиболее оптимального теоретического закона распределения скоростей ветра на юговостоке европейской территории России (ЕTP), включающей Астраханскую, Волгоградскую, Оренбургскую, Саратовскую, Пензенскую, Самарскую, Ульяновскую области и Татарстан. К исследованию привлечены нормальный и логнормальный законы распределения, а также Максвелла и Вейбулла-Гудрича, применяемые для выравнивания ветрового режима различных территорий. В качестве исходных данных использовались многолетние данные о повторяемости скоростей ветра по градациям, содержащиеся в «Справочнике по климату СССР» [13] и «Научно-прикладном справочнике по климату СССР» [18]. Параметры, входящие в теоретические распределения, рассчитывались по этим данным, что влекло снижение степеней свободы для определения критического значения $\chi^{2}$ Пирсона в соответствии с уравнением (10).

Для оценки критериального значения $\chi^{2}$ Пирсона важно было оценить число степеней свободы, зависящее не только от количества градаций, но и от дополнительных соотношений. При выравнивании распределения скоростей ветра по нормальному и логнормальному законам число дополнительных соотношений $l=2$, по закону Максвелла $l=1$, а по закону Вейбулла-Гудрича $l=3$.

Как показали расчеты (табл. 1), по критерию $\chi^{2}$ нормальный и логнормальный законы распределения нельзя применять к выравниванию ветрового режима на рассматриваемой территории. Расчетные значения критерия Пирсона существенно выше критического, и, следовательно, гипотезы о том, что распределение скоростей ветра подчиняется этим законам, должны быть отвергнуты. Для законов распределения Максвелла и Вейбулла-Гудрича расчетные значения $\chi^{2}$ существенно ниже критических $\chi_{0}^{2}$. Поэтому можно считать, что по данному критерию для выравнивания скоростного режима ветра можно использовать указанные законы. Отметим, что значения $\chi^{2}$ для упомянутых законов распределения в несколько раз меньше критического $\chi_{0}^{2}=16,9$, а это повышает надежность принятия достоверной гипотезы. Расчеты выполнены для всех метеостанций юго-востока ЕТР, но ввиду громоздкости таблиц и аналогичности результатов в табл. 1 приведены значения критерии Пирсона лишь для метеостанций Пензенской области. 
Критерии согласия Пирсона $\chi^{2}$ по метеостанциям Пензенской области

\begin{tabular}{|l|c|c|c|c|c|c|c|c|c|c|c|c|c|}
\hline \multicolumn{1}{|c|}{ Станция } & I & II & III & IV & V & VI & VII & VIII & IX & X & XI & XII & Год \\
\hline \multicolumn{10}{|c|}{ Закон Максвелла } \\
\hline Анучино & 5,3 & 6,2 & 6,1 & 3,3 & 3,4 & 1,9 & 1,4 & 2,2 & 2,8 & 3,8 & 4,6 & 6,1 & 3,7 \\
\hline Земетчино & 4,3 & 4,5 & 4,0 & 2,5 & 3,4 & 2,5 & 2,5 & 4,2 & 4,0 & 3,7 & 3,3 & 4,5 & 3,8 \\
\hline Пенза & 4,8 & 4,4 & 5,2 & 4,0 & 3,9 & 3,4 & 3,1 & 3,3 & 3,6 & 5,0 & 4,2 & 5,0 & 3,9 \\
\hline \multicolumn{10}{|c|}{ Нормальный закон } \\
\hline Анучино & 15,7 & 16,2 & 16,8 & 14,0 & 14,3 & 13,0 & 10,9 & 11,7 & 14,9 & 15,2 & 15,3 & 17,9 & 13,6 \\
\hline Земетчино & 12,0 & 12,4 & 12,6 & 11,6 & 12,4 & 10,8 & 8,4 & 7,3 & 9,9 & 10,9 & 12,9 & 14,5 & 10,6 \\
\hline Пенза & 15,8 & 15,6 & 16,1 & 15,1 & 12,6 & 11,8 & 11,5 & 11,8 & 18,5 & 17,1 & 15,3 & 16,0 & 14,1 \\
\hline \multicolumn{10}{|c|}{ Логнормальный закон } \\
\hline Анучино & 25,2 & 23,5 & 25,9 & 21,0 & 27,6 & 20,2 & 18,0 & 22,1 & 27,8 & 28,7 & 29,0 & 32,2 & 25,5 \\
\hline Земетчино & 25,2 & 23,5 & 25,9 & 21,0 & 27,6 & 20,2 & 18,0 & 22,1 & 27,8 & 28,7 & 29,0 & 32,2 & 25,5 \\
\hline Пенза & 25,2 & 23,5 & 25,9 & 21,0 & 27,6 & 20,2 & 18,0 & 22,1 & 27,8 & 28,7 & 29,0 & 32,2 & 25,5 \\
\hline \multicolumn{10}{|c|}{ Закон Вейбулла-Гудрича } & \multicolumn{1}{c|}{} \\
\hline Анучино & 3,4 & 4,2 & 4,4 & 2,5 & 2,5 & 1,9 & 1,1 & 1,2 & 2,7 & 3,4 & 3,5 & 5,0 & 2,4 \\
\hline Земетчино & 2,0 & 2,4 & 2,1 & 1,6 & 2,1 & 1,0 & 0,5 & 0,3 & 0,9 & 1,4 & 2,2 & 3,2 & 1,3 \\
\hline Пенза & 3,9 & 3,8 & 4,1 & 3,0 & 2,4 & 1,7 & 1,2 & 1,3 & 3,6 & 4,2 & 3,9 & 4,0 & 2,7 \\
\hline
\end{tabular}

Критерий Колмогорова $\lambda$ сильно зависит от объема выборки, поскольку прямопропорционален $\sqrt{N}$. В «Справочнике по климату СССР» издания 1960-х гг. по большинству метеостанций таблица повторяемости скоростей ветра по градациям составлена в основном за период 1936-1960 гг., в это время измерения производились в основном по флюгеру 4 раза в сутки. Повторяемость конкретной градации скорости ветра представляет собой отношение суммы числа случаев определенных значений к общему числу наблюдений, включая штили. Данные получены непосредственно путем подсчета из ряда наблюдений срочные для каждого месяца и за год в целом. Поэтому объем выборки для конкретного месяца и года в целом определится произведением числа сроков наблюдений, числа дней в месяце или году и продолжительности расчетного периода. В «Научно-прикладном справочнике по климату СССР» издания 1980-х годов аналогичная таблица составлена за период 1966-1980 гг., когда наблюдения производились уже анеморумбометром
M-63 8 раз в сутки. В связи с этим при приблизительном равенстве периодов наблюдений объем выборки в данном издании почти в два раза больше и составил 43850 случаев за год, а корень из этого числа - чуть более 209. Погрешности расчета повторяемости скоростей ветра в градации составляют около $1 \%$. В связи с этим возникает ситуация, при которой даже при максимуме различия теоретической и эмпирической обеспеченностей на уровне погрешностей велика вероятность отвергнуть тот или иной приемлемый закон. Кроме того, относить к практически невозможным событиям 5\% случаев общего объема выборки нам представляется чрезмерным. Оправданнее расчеты производить для 1\%-го уровня значимости, для него $\lambda_{\text {крит }}=1,627$.

Как следует из табл. 2, для нормального, логнормального законов и для распределения Максвелла расчетные значения $\lambda$ на всех метеостанциях и во все месяцы и год в целом значительно выше критического. Лишь распределение Вейбулла-Гудрича не противоречит гипотезе соответствия эмпирическому скоростному режиму.

Критерии Колмогорова $\lambda$ для метеостанций Пензенской области

Таблица 2

\begin{tabular}{|l|c|c|c|c|c|c|c|c|c|c|c|c|c|}
\hline \multicolumn{1}{|c|}{ Станция } & I & II & III & IV & V & VI & VII & VIII & IX & X & XI & XII & Год \\
\hline \multicolumn{10}{|c|}{ Закон Максвелла } \\
\hline Анучино & 4,51 & 3,99 & 3,76 & 4,34 & 4,62 & 5,52 & 5,73 & 5,95 & 4,62 & 4,15 & 4,09 & 3,80 & 4,27 \\
\hline Земетчино & 4,19 & 4,19 & 4,46 & 4,32 & 3,87 & 4,89 & 6,62 & 5,93 & 4,97 & 5,16 & 4,03 & 3,95 & 4,41 \\
\hline Пенза & 3,00 & 2,59 & 2,16 & 2,12 & 2,07 & 4,09 & 4,33 & 4,24 & 3,18 & 2,28 & 2,51 & 1,95 & 2,93 \\
\hline \multicolumn{10}{|c|}{ Нормальный закон } \\
\hline Анучино & 4,76 & 4,61 & 4,51 & 4,59 & 4,59 & 4,51 & 5,12 & 4,93 & 4,11 & 4,42 & 4,59 & 4,26 & 4,89 \\
\hline
\end{tabular}


Окончание табл. 2

\begin{tabular}{|l|c|c|c|c|c|c|c|c|c|c|c|c|c|}
\hline \multicolumn{1}{|c|}{ Станция } & I & II & III & IV & V & VI & VII & VIII & IX & X & XI & XII & Год \\
\hline \multicolumn{10}{|c|}{ Нормальный закон } \\
\hline Земетчино & 5,79 & 5,51 & 5,41 & 5,18 & 5,12 & 5,12 & 6,10 & 6,87 & 5,70 & 5,59 & 4,94 & 4,62 & 5,84 \\
\hline Пенза & 4,42 & 4,20 & 4,43 & 4,43 & 5,30 & 5,04 & 4,93 & 4,85 & 3,45 & 4,18 & 4,35 & 4,50 & 4,71 \\
\hline \multicolumn{10}{|c|}{ Логнормальный закон } \\
\hline Анучино & 3,09 & 2,56 & 3,30 & 4,30 & 4,14 & 3,98 & 4,50 & 4,61 & 4,31 & 3,73 & 3,36 & 3,57 & 3,77 \\
\hline Земетчино & 3,41 & 3,60 & 3,13 & 3,69 & 4,02 & 3,79 & 4,13 & 3,35 & 3,80 & 0,71 & 3,85 & 3,70 & 3,63 \\
\hline Пенза & 4,09 & 2,07 & 3,86 & 4,47 & 3,06 & 2,49 & 3,61 & 3,84 & 4,84 & 4,27 & 4,19 & 4,61 & 4,16 \\
\hline \multicolumn{10}{|c|}{ Закон Вейбулла-Гудрича } & & & \\
\hline Анучино & 0,65 & 1,39 & 1,63 & 1,41 & 1,20 & 0,92 & 0,67 & 1,58 & 1,61 & 1,62 & 1,43 & 1,62 & 1,61 \\
\hline Земетчино & 0,25 & 1,50 & 0,15 & 1,39 & 0,48 & 0,55 & 0,30 & 1,53 & 1,00 & 1,56 & 1,61 & 1,22 & 1,38 \\
\hline Пенза & 1,59 & 1,56 & 1,30 & 1,29 & 0,49 & 1,20 & 1,53 & 0,34 & 1,54 & 1,51 & 1,08 & 1,40 & 1,50 \\
\hline
\end{tabular}

Принимая во внимание результаты испытания гипотез по двум критериям согласия, следует констатировать, что наиболее приемлемым теоретическим законом следует признать распределение Вейбулла-Гудрича, поэтому целесообразно использовать его для выравнивания реального ветрового режима. Материалы, представленные в данном исследовании, являются существенным дополнением к уже опубликованным в других работах характеристикам ветрового режима пограничного слоя атмосферы. Распределение Вейбулла-Гудрича может найти широкое применение при решении ряда практических задач, связанных с учетом особенностей распределения скоростей ветра в нижнем слое атмосферы. Важнейшими из таких задач являются расчеты, ветроэнергетических ресурсов территории, максимальных скоростей ветра различной периодичности, ветровых нагрузок на различные сооружения.

\section{Библиографический список}

1. Срезневский Б.И. О силе ветра в Петербурге и Кронштадте // Записки по гидрографии. 1889. Вып. 2. С. 15-23.

2. Кобымева Н.В., Гольберг М.А. Методические указания по статистической обработке метеорологических рядов. Л., $1990.83 \mathrm{c}$.

3. Гриневич Г.А. Основы энергетической характеристики режима ветра. М., 1963. С. 26-85.

4. Гриневич Г.А. Опыт разработки элементов малого ветроэнергетического кадастра Средней Азии и Казахстана. Ташкент, 1952. 152 с.

5. Брукс К., Карузерс Н. Применение статистических методов в метеорологии. Л., 1963. $416 \mathrm{c}$.

6. Гутерман И.Г. О статистическом законе распределения скоростей ветра // Метеорология и гидрология. 1961. № 9. C. 13-22.
7. Hesselberg und Byorkdal. Uber das Verteilungsgesetz der Windunruhe // Beitrage z. Physik. d. fr. Atm. 1929. Vol. 15. $121 \mathrm{p}$.

8. Кузнеиов Е.С. Закон распределения случайного вектоpa // ДАН СССР. II. № 3-4. 1935.

9. Оиманский М.A. Обработка наблюдений над ветром // Тр. ГГО. 1937. Вып. 18. Теоретическая метеорология (5).

10. Кошинский С.Д. Режимные характеристики сильных ветров на морях Советского Союза: В 2 ч. Ч. 1. Каспийское море. Л., 1975. 412 с.; Ч. 2. Север Японского, Охотское и Беренгово моря. Л., 1978. $391 \mathrm{c.}$

11. Кошинский С.Д. Из опыта расчета некоторых статистических характеристик ветра и параметров функции распределения вида $f(x)=\exp \left[-\left(\frac{v}{\beta}\right)^{\gamma}\right]$ на электронновычислительных машинах // Тр. НР ГМЦ СССР. 1969. Вып. 2. С. 44-53.

12. Брагинская Л.Л., Каган Р.Л. К вопросу об аппроксимации распределения скоростей ветра // Тр. ГГО. 1982. Вып. 447. С. 49-57.

13. Справочник по климату СССР: В 3 ч. Ч. 3. Л., 1967. Вып. 9.

14. Анапольская Л.Е. Режим скоростей ветра на территории СССР. Л., 1961. 199 с.

15. Justus C.G., Hazgraves W.R., Yalcin A. Nationwide assessment of potential output from wind-powered generators // J. Appl. Met. 1976. Vol. 15, № 7. P. 673-678.

16. Заварина М.B. Расчетные скорости ветра на высотах нижнего слоя атмосферы. Л., 1971. 162 с.

17. Анапольская Л.Е., Гандин Л.С. Ветроэнергетические ресурсы и методы их оценки // Метеорология и гидрология. 1978. № 7. С. 11-17.

18. Научно-прикладной справочник по климату СССР. Л., 1988. Сер. 3. Вып. 12-13. 